\title{
Sweater online customization system based on mobile terminal
}

\author{
Yanmei Liu \\ School of Art Design, Jiaxing University, Jiaxing Zhejiang China \\ yanmei0_lym@163.com
}

Keywords: mobile terminal; online customization system; sweater; Android OS

Abstract. The usage of sweater online customization system indicates that the system has broad application prospects. This paper takes Android system as a typical example and the online implementation system is developed. Besides, the key technologies of online sweater-designing system are introduced which includes data parsing, data feedback and image loading. Finally, the operating procedures and its advantages are briefly discussed in the following paragraphs.

\section{Introduction}

The apparel mass customization is a production mode which is adapted to market demand in recent years. The essential differences between this and the traditional way of single tailored clothing is that the former provides personalized products and services at low cost and high efficiency just the similar as the mass production way. The research of garment mass customization has been extended from the concept and mode to business process and technology. Wang et al. [1] discussed the promoting function of the electronic commerce to costume mass customization, analyzed several marketing model of garment mass customization under the era of electronic commerce. Guo et al. [2] designed garment mass customization system based on the construction of knowledge base of enterprise production resource, which embodied modular design thought. These studies involved the design and development of garment mass customization under the environment of e- commerce, but very few applications are based on mobile terminals[3].

34 times China Internet network development state statistic report[4], released by CNNIC, shows that, up to June 2014, in the online devices used by netizens, cell phone usage is up to 83.4 percent, which is beyond the traditional PC overall utilization for the first time( 80.9 percent). In the first half of 2015, mobile payment, mobile shopping, mobile travel reservation, user scale is up to 276 million, 276 million and 270 million, respectively[5]. In the face of such a large mobile terminal users and the market, mobile business will be the main market in the future. The newest report of Strategy Analytic shows that, smartphone shipments 320 million units in the third quarter of 2014, Android has 84 percent[6].

In the face of mobile terminal users demand, the development of sweater mass customization system based on mobile terminal to the transition of the traditional sweater industry production mode and upgrade has very important significance. Therefore, a sweater online customization system based on the Android OS, which is the most representative of mobile OS, is designed in this article.

In the following several sections, the key technologies of sweater online customization system based on mobile terminal is introduced, System development process and system implementation GUI are described, then, the technical advantages of this system are given, and finally, a brief summary is provided. 


\section{Key Technologies of Sweater Online Customization System}

Data Parsing. The filtered data returns to the client from the server in XML form. So in Android client, data parsing for XML is necessary to list the data returned from the server to supply convenience for later use. In Android OS, there are three kinds of XML parser, including the DOM parser, SAX parser and PULL parser. PULL parser is small and light, simple and easy to use, which has high parsing speed, and is very suitable for use in Android mobile devices. In the interior of Android OS, PULL parser is used when parsing all kinds of XML. The operating principle of PULL parser is: XML PULL provides a start element and the end element; when an element beginning, the parser.NextText is invoked to extract all character data from the XML document; at the end of a document, the EndDocument events will be generated automatically.

For the type of request data is different, the return data structure is also different. By writing the base class XmlPullParserBase of PULL parse, the class is called when data analysis is needed, and data will be putted into different Bean object according to different nodes, which makes full use of the flexibility of PULL parser. When the system requests data, it will call a getList() method inheriting from XmlPullParserBase class object, then send the requested URL connection. According to different nodes, different variables of the Bean object will put into the XML data, which returns through the PULL parser parsing server. When parsing a node, a Bean object will put into a list. If the process of parsing is over, the whole parsing process is completed.

Data Feedback. Data feedback is carried out through XML data format. Because there are several kinds of XML parser, the method of data feedback using XML will take convenient to let the data received by the client. For the reason that XML data format is standard, it can be handled conveniently in the client. Furthermore, XML can be directly displayed on a web page, so can be accessed directly through the URL during debugging, and then to check the data in XML. So in the development process, server is separated from client, independence is higher.

Taking component information obtaining as an example, the following is the core program of pseudo code.

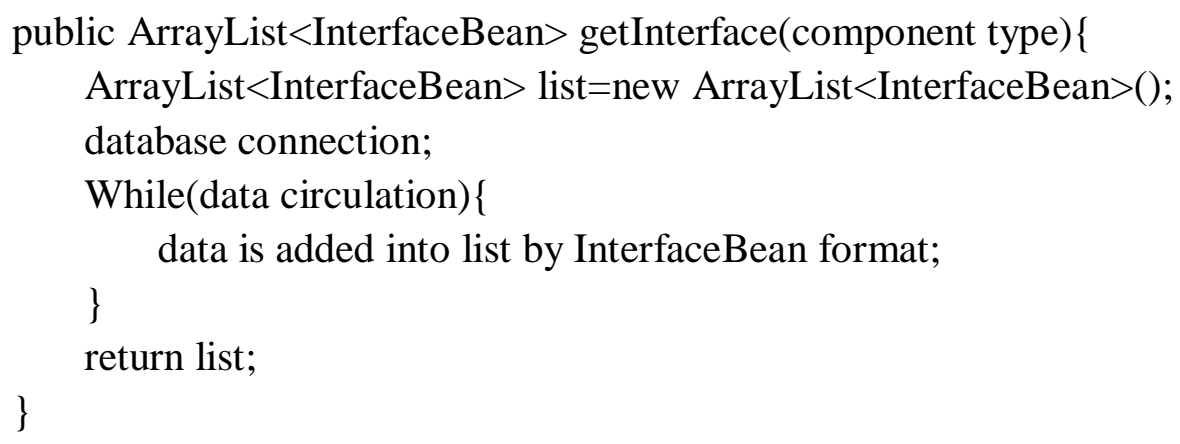

Image Loading. During system running, variety network images with different size are needed to load, such as component product images, module images, fabric images and so on. The setMemCacheSizePercen() method of the utility class ImageCache is used to set an image storage space with the specified size. When the network image is firstly accessed, the image is put into the space. Every time an image is requested, the first thing is to check that whether the image resource has been loaded in the cache. Load the image directly if it already exists; on the other hand, download it from the network, and put it into the cache. Because cache has certain release speed, and a single UI does not need to load a lot of images in the system, there will be no buffer overflow.

The class ImageFetcher is used to load image. In ImageFetcher class, there is an image loading method loadImage(), which can put the obtained image into the container only by passing the parameter of image URL and the image display container ImageView. 
Except accessing to the network images, the system will also load the local images. When a user wants to his favorite pattern add to sweater customization, the system allows the user to load some local images. To load local image, the first step is reading the file to output stream, and then use the BitmapFactory.DecodeByteArray() method to form a Bitmap object and put it into the image container. The following is the core program of pseudo code.

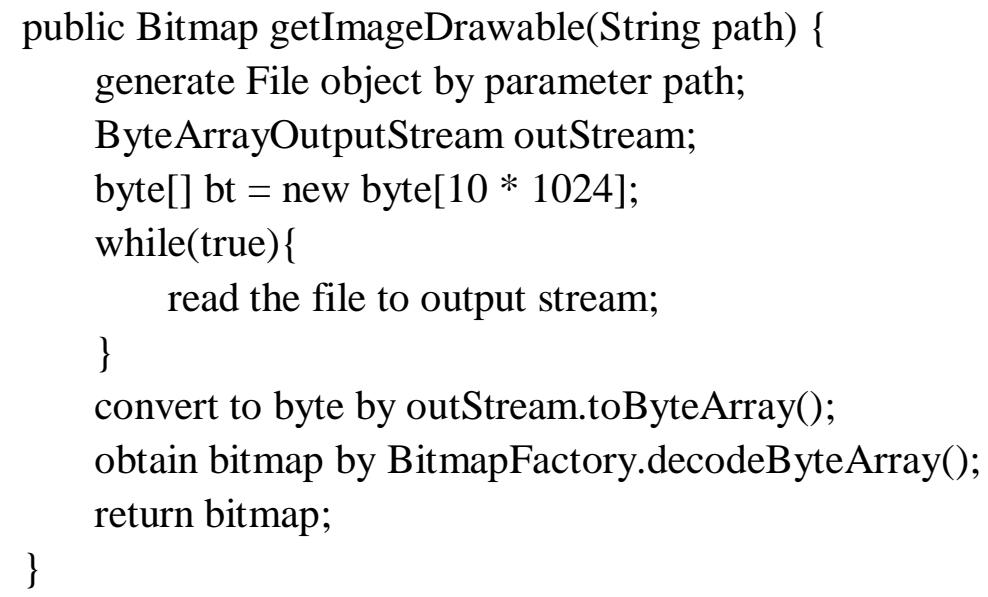

\section{System Implementation}

The system is divided into client subsystem and server subsystem. The whole process of system operation is shown as figure 1. We focus on client subsystem, which gears to the needs of customers. Customers can choose their favorite sweater styles in the provided products library, or choose elements in fabric library, process library. When the user wants to design an individualization product, if he knows his body measurement, he can select components from module library, such as clothes body, sleeve, neckband, color, texture, decorative, and so on. All of user's selection needs to be satisfied constraint rules. All of the selected modules will be form a customized sweater and can be showed on the torso model, which will vivid display. What's more, the system supports online order generation and online payment. System GUI is shown as figure 2.

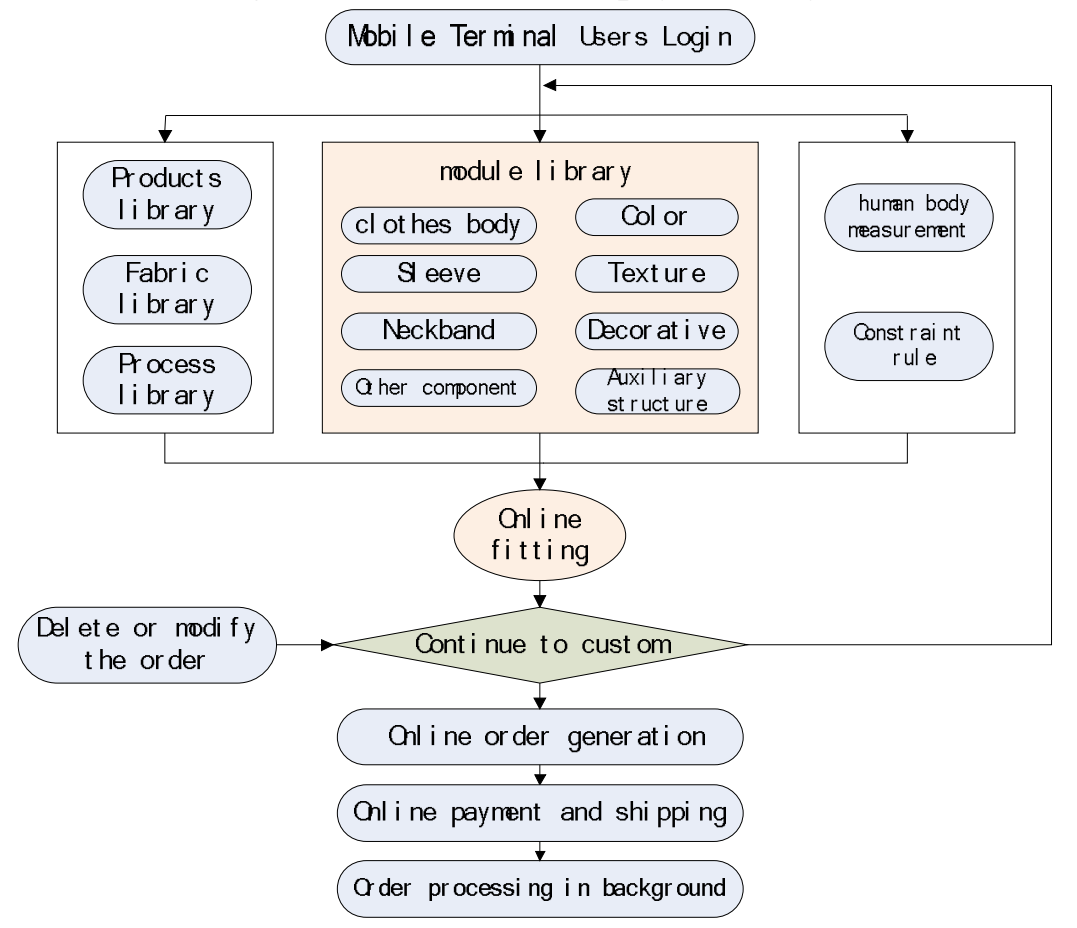

Fig.1. System operation process 


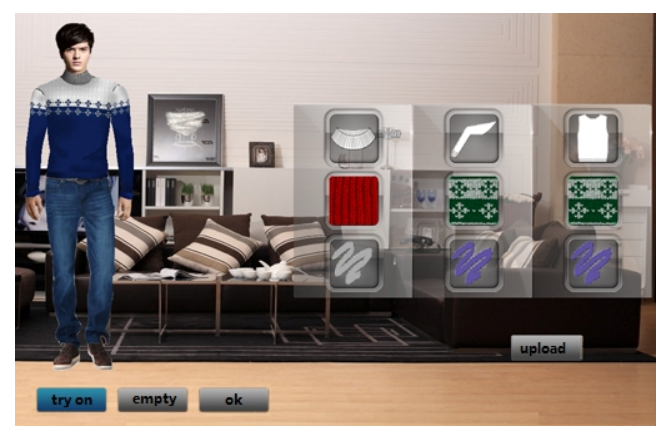

Fig.2. System GUI

\section{System Advantages}

The main advantage of this system is based on the combination of components customized function. Users can select components from module library to form a customized sweater which absolutely accords with his body measurement.

The second advantage of this system can run on mobile terminal, especially on Android OS, so users can directly complete sweater custom in Android devices, such as mobile phone, tablet PC, set-top boxes and other Android devices. The superiority of mobile devices is that they can custom and order anytime and anywhere.

\section{Conclusion}

At present, online customization system based on mobile terminal is rare, so the sweater customization system designed in this paper has good demonstration significance. Key technologies of sweater online customization system based on mobile terminal are introduced, core pseudo code of data feedback and image loading are described, system implementation process is given, which can provide a reference for similar application development.

\section{Acknowledgments}

This work was supported by Scientific Research Program of the Education Department of Zhejiang Province, China (No.Y201533781).

\section{References}

[1] Wang qian, Zhou jianjun. Analysis on Marketing Mode of Garment Mass Customization in the Trend of Electronic Commerce[J].China Textile Leader,2014(5):118-121.

[2] Guo lizi, Wu Jianyi. A Study on Apparel Mass Customization System Based on Knowledge Base of Production Resources[J].China Textile Leader,2013(12):76-78.

[3] Dai Weihui.Mass customization supply chain research in mobile environment[D]. Shanghai: Fudan University,2009.

[4] 34 times China Internet network development state statistic report[EB/OL]. http://www.cnnic. net.cn/hlwfzyj/hlwxzbg/hlwtjbg/201407/P020140721507223212132.pdf.

[5] 36 times China Internet network development state statistic report[EB/OL]. http://www.cnnic. net.cn/ hlwfzyj/hlwxzbg/ hlwtjbg/201507/P020150723549500667087.pdf.

[6] Strategy Analytics. Smartphone shipments 320 million units in the third quarter of 2014, Android has 84 percent[EB/OL]. http://www.c114.com.cn/news/116/a866270.html 